\title{
Fixed Point Theorems for Noncyclic Monotone Relatively $\rho$-Nonexpansive Mappings in Modular Spaces
}

\author{
Nour-eddine El Harmouchi $\left(\mathbb{D}\right.$, Karim Chaira ${ }^{(D)}$, and El Miloudi Marhrani ${ }^{\circ}$ \\ Laboratory of Algebra Analysis and Applications, Hassan II University of Casablanca, Faculty of Sciences Ben M'Sik, \\ Avenue Driss El Harti, B. P 7955, Sidi Othmane, Casablanca, Morocco
}

Correspondence should be addressed to El Miloudi Marhrani; marhrani@gmail.com

Received 21 September 2019; Accepted 6 May 2020; Published 22 June 2020

Academic Editor: Luca Vitagliano

Copyright (c) 2020 Nour-Eddine El Harmouchi et al. This is an open access article distributed under the Creative Commons Attribution License, which permits unrestricted use, distribution, and reproduction in any medium, provided the original work is properly cited.

In this paper, we obtain some fixed point results for noncyclic monotone $\rho$-nonexpansive mappings in uniformly convex modular spaces and uniformly convex in every direction modular spaces. As an application, we prove the existence of the solution of an integral equation.

\section{Introduction}

The notion of modular spaces, as a generalization of Banach spaces, was firstly introduced by Nakano [1] in connection with the theory of ordered spaces. These spaces were developed and generalized by Orlicz and Musielak [2].

It is well known that fixed point theory is an active field of research, a powerful tool in solving integral and differential equations. Following the publications of Ran and Reuring [3] and Neito and Rrodriguez-Lopez [4], fixed point theory in partially ordered modular spaces has recently received a good attention from researchers.

Alfuraidan et al. [5] gave a modular version of Ran and Reuring fixed point theorem and proved the existence of fixed point of monotone contraction mappings in modular function spaces. Thereafter, they gave an extension of their main results for pointwise monotone contractions. In [6], Gordji et al. have proved that any quasi-contraction mappings in partially ordered modular spaces without $\Delta_{2}$-condition have a fixed point.

In 2016, Bin Dehaish and Khamsi proved in [7] the following theorems.

Theorem 1 (see [7]). Let $\rho \in \mathscr{R}$ be a (UUC1) and $C$ be a nonempty convex $\rho$-closed $\rho$-bounded subset of $L_{\rho}$ not reduced to one point. Let $T: C \longrightarrow C$ be a monotone $\rho$-nonexpansive mapping and $\rho$-continuous. Assume there exists $f_{0} \in C$ such that $f_{0}$ and $T f_{0}$ are comparable. Then, $T$ has a fixed point.

Theorem 2 (see [7]). Assume that $\rho \in \mathscr{R}$ is UUCED and uniformly continuous. Assume that $L_{\rho}$ satisfies the property (R). Let $C$ be a nonempty convex $\rho$-closed $\rho$-bounded subset of $L_{\rho}$ not reduced to one point. Let $T: C \longrightarrow C$ be a monotone $\rho$-nonexpansive mapping and $\rho$-continuous. Assume there exists $f_{0} \in C$ such that $f_{0}$ and $T f_{0}$ are comparable. Then, $T$ has a fixed point.

These theorems are a generalization to modular function spaces of Browder and Göhde fixed point theorem for monotone nonexpansive mappings in Banach spaces.

A mapping $T: A \cup B \longrightarrow A \cup B$ is said to be noncyclic provided that $T(A) \subseteq A$ and $T(B) \subseteq B$, where $(A, B)$ is a nonempty pair in a modular space. When we consider this type of mappings, it is interesting to ask if it is possible to find a pair $\left(x^{*}, y^{*}\right) \in A \times B$ such that

$$
\begin{aligned}
T x^{*} & =x^{*}, \\
T y^{*} & =y^{*}, \\
\rho\left(x^{*}-y^{*}\right) & =d_{\rho}(A, B),
\end{aligned}
$$

that is, $\left(x^{*}, y^{*}\right)$ is a best proximity pair. 
In this paper, we discuss some best proximity pair results for the class of monotone noncyclic relatively $\rho$-nonexpansive mappings in the framework of modular spaces equipped with a partial order defined by a $\rho$-closed convex cone $P$, that is, for any $x$ and $y$ in the modular space $X_{\rho}$ one has $x \preceq y$ if and only if $y-x$ in $P$. We took this ordering because in the case of modular spaces the order intervals are not convex closed as in modular function spaces (see Theorem 2.4 in [7]). Our results generalize Theorems 1 and 2 and others for monotone $\rho$-nonexpansive mappings to the case of noncyclic monotone relatively $\rho$-nonexpansive mappings in modular spaces.

To illustrate the effectiveness of our main results, we give an application to an integral equation which involves noncyclic monotone $\rho$-nonexpansive mappings.

\section{Materials and Methods}

The materials used in this study are obtained from journal articles and books existing on the Internet. The main tools used in this manuscript are the uniform convexity and the uniform convexity in every direction of a modular $\rho$. The aim of this work is to extend some fixed point theorems for nonexansive mappings to best proximity pair for monotone noncyclic relatively $\rho$-nonexpansive mappings in modular spaces.

The paper is organized as follows. In Section 3, we begin with recollection of some basic definitions and lemmas with corresponding references that will be used in the sequel. Thereafter, we give the main results of the paper with some examples. To validate the utility of our results, we give in Section 4 an application to an integral equation.

\section{Preliminaries}

Throughout this work, $X$ stands for a linear vector space on the field $\mathbb{R}$. Let us start with some preliminaries and notations.

Definition 1 (see [8]). A function $\rho: X \longrightarrow[0,+\infty]$ is called a modular if the following holds:

(1) $\rho(x)=0$ if and only if $x=0$

(2) $\rho(-x)=\rho(x)$

(3) $\rho(\alpha x+(1-\alpha) y) \leq \rho(x)+\rho(y)$, for any $\alpha \in[0,1]$ and for any $x$ and $y$ in $X$

If (3) is replaced by $\left(3^{\prime}\right) \rho(\alpha x+(1-\alpha) y) \leq \alpha \rho(x)+(1-$ $\alpha) \rho(y)$ for any $\alpha \in[0,1]$ and $x$ and $y$ in $X$, then $\rho$ is called a convex modular.

The modular space is defined as $X_{\rho}=\left\{x \in X: \lim _{\lambda \longrightarrow 0} \rho(\lambda x)=0\right\}$. Throughout this paper, we will assume that the modular $\rho$ is convex. The Luxemburg norm in $X_{\rho}$ is defined as

$$
\|x\|_{\rho}=\inf \left\{\lambda>0: \rho\left(\frac{x}{\lambda}\right) \leq 1\right\} .
$$

Associated to a modular, we introduce some basic notions needed throughout this work.
Definition 2 (see [8]). Let $\rho$ be a modular defined on a vector space $X$ :

(1) We say that a sequence $\left(x_{n}\right)_{n \in \mathbb{N}} \subset X_{\rho}$ is $\rho$-convergent to $x \in X_{\rho}$ if and only if $\lim _{n \longrightarrow \infty} \rho\left(x_{n}-x\right)=0$. Note that the limit is unique.

(2) A sequence $\left(x_{n}\right)_{n} \subset X_{\rho}$ is called $\rho$-Cauchy if $\rho\left(x_{n}-\right.$ $\left.x_{m}\right) \longrightarrow 0$ as $n, m \longrightarrow+\infty$.

(3) We say that $X_{\rho}$ is $\rho$-complete if and only if any $\rho$-Cauchy sequence is $\rho$-convergent.

(4) A subset $C$ of $X_{\rho}$ is said $\rho$-closed if the $\rho$-limit of a $\rho$-convergent sequence of $C$ always belong to $C$.

(5) A subset $C$ of $X_{\rho}$ is said $\rho$-bounded if we have $\delta_{\rho}(C)=\sup \{\rho(x-y): x, y \in C\}<\infty$.

(6) A subset $K$ of $X_{\rho}$ is said $\rho$-sequentially compact if any sequence $\left(x_{n}\right)_{n}$ of $C$ has a subsequence $\rho$-convergent to a point $x \in C$.

(7) We say that $\rho$ satisfies the Fatou property if $\rho(x-$ $y) \leq \lim _{n \longrightarrow+\infty} \rho\left(x-y_{n}\right) \quad$ whenever $\quad\left(y_{n}\right)_{n} \rho$-converges to $y$ for any $x, y$, and $y_{n}$ in $X_{\rho}$.

Let us note that $\rho$-convergence does not imply $\rho$-Cauchy condition. Also, $x_{n} \stackrel{\rho}{\longrightarrow} x$ does not imply in general $\lambda x_{n} \stackrel{\rho}{\longrightarrow} \lambda x$, for every $\lambda>1$.

Definition 3. Let $\rho$ be a modular and $C$ be a nonempty subset of the modular space $X_{\rho}$. A mapping $T$ : $C \preceq C$ is said to be

(a) Monotone, if $T(x) \preceq T(y)$ for any $x, y \in C$ such that $x \preceq y$.

(b) Monotone $\rho$-nonexpansive, if $T$ is monotone and

$$
\rho(T(x)-T(y)) \preceq \rho(x-y),
$$

whenever $x, y \in X_{\rho}$ and $x \preceq y$.

Recall that $T: C \longrightarrow C$ is said to be $\rho$-continuous if $\left(T\left(x_{n}\right)\right)_{n} \rho$-converges to $T(x)$ whenever $\left(x_{n}\right)_{n} \rho$-converges to $x$. It is not true that a monotone $\rho$-nonexpansive mapping is $\rho$-continuous, since this result is not true in general when $\rho$ is a norm.

Let $A$ and $B$ be nonempty subsets of a modular space $X_{\rho}$. We adopt the notation $d_{\rho}(A, B)=\inf \{\rho(x-y)$ : $x \in A, y \in B\}$.

A pair $(A, B)$ is said to satisfy a property if both $A$ and $B$ satisfy that property. For instance, $(A, B)$ is $\rho$-closed (resp. convex, $\rho$-bounded) if and only if $A$ and $B$ are $\rho$-closed (resp. convex, $\rho$-bounded). A pair $(A, B)$ is not reduced to one point means that $A$ and $B$ are not singletons.

Recall the definition of the modular uniform convexity.

Definition 4 (see [8]). Let $\rho$ be a modular and $r>0$ and $\varepsilon>0$. Define, for $i \in\{1,2\}$,

$$
D_{i}(r, \varepsilon)=\left\{(x, y) \in X_{\rho} \times X_{\rho}: \rho(x) \leq r, \rho(y) \leq r, \rho\left(\frac{x-y}{i}\right) \geq r \varepsilon\right\} .
$$




$$
\delta_{i}(r, \varepsilon)=\inf \left\{1-\frac{1}{r} \rho\left(\frac{x+y}{2}\right):(x, y) \in D_{i}\right\} .
$$

If $D_{i}(r, \varepsilon)=\varnothing$, we set $\delta_{i}(r, \varepsilon)=1$.

(i) We say that $\rho$ is uniformly convex (UCi) if for every $r>0$ and $\varepsilon>0$, and we have $\delta_{i}(r, \varepsilon)>0$

(ii) We say that $\rho$ is unique uniformly convex (UUCi) if for all $s \geq 0$ and $\varepsilon>0$, and there exists $\eta(s, \varepsilon)>0$ such that $\delta_{i}(r, \varepsilon)>\eta(s, \varepsilon)$, for $r>s$

(iii) We say that $\rho$ is strictly convex (SC), if for every $x$, $y \in X_{\rho} \quad$ such that $\rho(x)=\rho(y)$ and $\rho((x+y) / 2)=\rho(x)+\rho(y) / 2$, and we have $x=y$

The following proposition characterizes the relationship between the above notions

Proposition 1 (see [8]).

(a) (UUCi) implies (UCi) for $i=1,2$

(b) $\delta_{1}(r, \varepsilon) \leq \delta_{2}(r, \varepsilon)$ for $r>0$ and $\varepsilon>0$

(c) (UC1) implies (UC2) implies (SC)

(d) (UUC1) implies (UUC2)

Definition 5 (see [9]). Let $\rho$ be a modular. We say that the modular space $X_{\rho}$ satisfies the property $(R)$ if and only if for every decreasing sequence $\left(C_{n}\right)_{n \in \mathbb{N}}$ of nonempty $\rho$-closed convex and $\rho$-bounded subsets of $X_{\rho}$ has a nonempty intersection.

Lemma 1 (see [8]). Let $\rho$ be a convex modular satisfying the Fatou property. Assume that $X_{\rho}$ is $\rho$-complete and $\rho$ is $\left(U U C_{2}\right)$. Then, $X_{\rho}$ satisfies the property $(R)$.

Definition 6 (see [8]). Let $\left(x_{n}\right)_{n}$ be a sequence in $X_{\rho}$ and $K$ be a nonempty subset of $X_{\rho}$. The function $\tau: K \longrightarrow[0, \infty]$ defined by $\tau(x)=\lim \sup _{n \longrightarrow \infty} \rho\left(x_{n}-x\right)$ is called a $\rho$-type function.

Note that the $\rho$-type function $\tau$ is convex since $\rho$ is convex. A sequence $\left(c_{n}\right)_{n} \subset K$ is called a minimizing sequence of $\tau$ if $\lim _{n \longrightarrow+\infty} \tau\left(c_{n}\right)=\inf _{x \in K} \tau(x)$.

The following result, found in [8], plays a crucial role in the proof of many fixed point results in modular spaces.

Lemma 2 (see [8]). Let $\rho$ be a convex modular (UUC1) satisfying the Fatou property and $X_{\rho}$ a $\rho$-compete modular space. Let $C$ be a nonempty $\rho$-closed convex subset of $X_{\rho}$. Consider the $\rho$-type function $\tau: C \longrightarrow[0,+\infty]$ generated by a sequence $\left(x_{n}\right)_{n}$ in $X_{\rho}$. Assume that $\tau_{0}=\inf \{\tau(x): x \in C\}<\infty$. Then, all the minimizing sequences of $\tau$ are $\rho$-convergent to the same limit.

\section{Main Results}

A subset $P \subset X_{\rho}$ is a pointed $\rho$-closed convex cone, if $P$ is a nonempty $\rho$-closed subset of $X_{\rho}$ satisfying the following properties:

(i) $P+P \subset P$

(ii) $\lambda P \subset P$, for all $\lambda \in \mathbb{R}_{+}$

(iii) $P \cap(-P)=\{0\}$

Using $P$, we define an ordering on $X_{\rho}$ by $x \preceq y$ if and only if $y-x \in P$.

Let $\rho$ be a convex modular and $T: C \longrightarrow C$ be a monotone mapping, where $C$ is a nonempty convex subset of the modular space $X_{\rho}$. Let $x_{0} \in C$ and $\lambda \in(0,1)$. Consider Krasnoselskii-Ishikawa iteration sequence $\left(x_{n}\right)_{n \in \mathbb{N}}$ in $C$ defined by $x_{n+1}=(1-\lambda) x_{n}+\lambda T\left(x_{n}\right)$ for all $n \in \mathbb{N}$. Assume that $x_{0} \preceq T\left(x_{0}\right)$ (resp. $\left.T\left(x_{0}\right) \preceq x_{0}\right)$. By the definition of the partial ordering $\preceq$, we obtain $x_{0} \preceq x_{1} \preceq T\left(x_{0}\right)$ (resp. $\left.T\left(x_{0}\right) \preceq x_{1} \preceq x_{0}\right)$.

Since $T$ is monotone, one has $T\left(x_{0}\right) \preceq T\left(x_{1}\right)$ (resp. $\left.T\left(x_{1}\right) \preceq T\left(x_{0}\right)\right)$. By induction, we prove that

$$
\begin{aligned}
x_{n} & \preceq x_{n+1} \preceq T\left(x_{n}\right) \preceq T\left(x_{n+1}\right)\left(\operatorname{resp} . T\left(x_{n+1}\right) \preceq T\left(x_{n}\right)\right. \\
& \left.\preceq x_{n+1} \preceq x_{n}\right), \quad \text { for all } n \in \mathbb{N} .
\end{aligned}
$$

Let us introduce the class of mappings for which the problem of fixed point will be considered.

Definition 7. Let $(A, B)$ be a nonempty pair of a modular space $X_{\rho}$. A mapping $T: A \cup B \longrightarrow A \cup B$ is said to be monotone noncyclic relatively $\rho$-nonexpansive if it satisfies the following conditions:

(i) For all $(x, y) \in(A \cup B)^{2}, x \preceq y$ implies that $T x \preceq T y$

(ii) $T(A) \subseteq A$ and $T(B) \subseteq B$

(iii) For all $x \in A$ and $y \in B$ such that $x \preceq y$, we have $\rho(T x-T y) \leq \rho(x-y)$

Definition 8 (see [10]). Let $(A, B)$ be a pair of a modular space $X_{\rho}$ and $T: A \cup B \longrightarrow A \cup B$ be a noncyclic mapping. A pair $(x, y) \in A \times B$ is said to be a best proximity pair for the noncyclic mapping $T$ if

$$
\begin{aligned}
T x & =x, \\
T y & =y, \\
\rho(x-y) & =d_{\rho}(A, B) .
\end{aligned}
$$

Definition 9. A space $X_{\rho}$ is said to satisfy the property $(p)$, if $x_{n} \stackrel{\rho}{\longrightarrow} x$ and $y_{n} \stackrel{\rho}{\longrightarrow} y$ with $x_{n} \preceq y_{n}$ for all $n \in \mathbb{N}$; then, $x \preceq y$.

We use $\left(A_{0}^{\leq}, B_{0}^{\leq}\right)$to denote the ordered $\rho$-proximal pair obtained from $(A, B)$ by

$$
\begin{aligned}
& A_{0}^{\leq}=\left\{x \in A: \rho(x-y)=d_{\rho}(A, B) \text { and } x \preceq y \text { for some } y \in B\right\} \\
& B_{0}^{\leq}=\left\{y \in B: \rho(x-y)=d_{\rho}(A, B) \text { and } x \preceq y \text { for some } x \in A\right\} .
\end{aligned}
$$


Note that if $A_{0}^{\leq}$is nonempty if and only if $B_{0}^{\leq}$is nonempty. Indeed, if $x_{0} \in A_{0}^{\leq}$then there exists $y \in B$ such that $x_{0} \preceq y$ and $\rho\left(x_{0}-y\right)=d_{\rho}(A, B)$. Thus, for this $y$ we take $x=x_{0} \in A$, we have $x \preceq y$ and $\rho(x-y)=d_{\rho}(A, B)$, that is, $y \in B_{0}^{\leq}$. As the same we prove the converse.

Proposition 2. Let $\rho$ be a convex modular satisfying the Fatou property and $X_{\rho}$ satisfies the property $(P)$. Let $(A, B)$ be a nonempty convex pair of $X_{\rho}$. Assume that $A_{0}^{\leq}$is nonempty:

(i) If $(A, B)$ is $\rho$-sequentially compact, then $\left(A_{0}^{\leq}, B_{0}^{\leq}\right)$is $\rho$-sequentially compact

(ii) $\left(A_{0}^{\leq}, B_{0}^{\leq}\right)$is convex

(iii) $d_{\rho}\left(A_{0}^{\leq}, B_{0}^{\leq}\right)=d_{\rho}(A, B)$

(iv) If $T: C \longrightarrow C$ is a noncyclic monotone relatively $\rho$-nonexpansive mapping, then $T\left(A_{0}^{\leq}\right) \subseteq A_{0}^{\leq}$and $T\left(B_{0}^{\leq}\right) \subseteq B_{0}^{\leq}$, that is, $A_{0}^{\leq}$and $B_{0}^{\leq}$are $T$-invariant

Proof. It is quite easy to verify (iii):

(i) Let $\left(x_{n}\right)_{n}$ be a sequence in $A_{0}^{\leq}$. Then, there exists a sequence $\left(y_{n}\right)_{n}$ in $B$ such that $x_{n} \preceq y_{n}$ and $\rho\left(x_{n}-\right.$ $\left.y_{n}\right)=d_{\rho}(A, B)$ for all $n \geq 0$. Since $(A, B)$ is $\rho$-sequentially compact, there exists subsequences $\left(x_{n_{k}}\right)_{k}$ and $\left(y_{n_{k}}\right)_{k}$ of $\left(x_{n}\right)_{n}$ and $\left(y_{n}\right)_{n}$, respectively, such that $x_{n_{k}} \preceq y_{n_{k}}$ for all $k \geq 0$, and $\rho$-converge to $x \in A$ and $y \in B$, respectively.

From property $(P)$, one has $x \preceq y$. Since $\rho$ satisfies the Fatou property, then

$d_{\rho}(A, B) \leq \rho(x-y) \leq \liminf _{k \longrightarrow \infty} \rho\left(x_{n_{k}}-y_{n_{k}}\right)=d_{\rho}(A, B)$.

Thus, $\rho(x-y)=d_{\rho}(A, B)$. Therefore, $A_{0}^{\leq}$is $\rho$-sequentially compact. Likewise, we prove that $B_{0}^{\leq}$is also $\rho$-sequentially compact.

(ii) Let $x_{1}, \quad x_{2} \in A_{0}^{\leq}, \quad \delta \in(0,1) \quad$ and set $z=\delta x_{1}+(1-\delta) x_{2}$. Since $x_{1}, x_{2} \in A_{0}^{\leq}$, then there exists $y_{1}, y_{2} \in B$ such that $x_{1} \preceq y_{1}, x_{2} \preceq y_{2}$ and $\rho\left(x_{1}-y_{1}\right)=\rho\left(x_{2}-y_{2}\right)=d_{\rho}(A, B)$. Moreover, $\delta x_{1} \preceq \delta y_{1} \quad$ and $\quad(1-\delta) x_{2} \preceq(1-\delta) y_{2}$. Hence, $z=\delta x_{1}+(1-\delta) x_{2} \preceq z \prime=\delta y_{1}+(1-\delta) y_{2}$. Since $\rho$ is convex, then

$$
\begin{aligned}
d_{\rho}(A, B) & \leq \rho\left(z-z^{\prime}\right)=\rho\left(\left(\delta x_{1}+(1-\delta) x_{2}\right)-\left(\delta x_{1}+(1-\delta) x_{2}\right)\right) \\
& \leq \delta \rho\left(x_{1}-y_{1}\right)+(1-\delta) \rho\left(x_{2}-y_{2}\right) \\
& \leq \delta d_{\rho}(A, B)+(1-\delta) d_{\rho}(A, B)=d_{\rho}(A, B) .
\end{aligned}
$$

Then, $\quad \rho\left(z-z^{\prime}\right)=d_{\rho}(A, B)$. Hence, $\quad z \in A_{0}^{\leq}$. Therefore, $A_{0}^{\leq}$is convex. Using the same argument we prove that $B_{0}^{\leq}$is convex.

(iii) Let $x \in A_{0}^{\leq}$, then there exists $y \in B$ such that $x \preceq y$ and $\rho(x-y)=d_{\rho}(A, B)$. Since $T$ is monotone noncyclic relatively $\rho$-nonexpansive, then $T x \preceq T y$ and $d_{\rho}(A, B) \leq \rho(T x-T y) \leq \rho(x-y)=d_{\rho}(A, B)$. Thus, $\rho(T x-T y)=d_{\rho}(A, B)$. Therefore, $T x \in A_{0}^{\leq}$. As the same way, we obtain $T\left(B_{0}^{\leq}\right) \subseteq B_{0}^{\leq}$.

Theorem 3. Let $\rho$ be a convex modular (UUC1) satisfying the Fatou property and $X_{\rho}$ be a $\rho$-complete modular space. Let $(A, B)$ be a nonempty convex and $\rho$-bounded pair of $X_{\rho}$ such that the subset $B_{0}^{\leq}$is nonempty $\rho$-closed. Let $T: A \cup B \longrightarrow A \cup B$ be a monotone noncyclic relatively $\rho$-nonexpansive mapping and $\rho$-continuous on $B$. Assume that there exists $x_{0} \in A_{0}^{\preceq}$ such that $x_{0} \preceq T x_{0}$. Then, $T$ has a best proximity pair.

Proof. Let $x_{0} \in A_{0}^{\leq}$such that $x_{0} \preceq T x_{0}$. Consider the sequence $\left(x_{n}\right)_{n}$ defined by $x_{n+1}=\lambda T x_{n}+(1-\lambda) x_{n}$ for all $n \geq 0$ and $\lambda \in(0,1)$. Set $C_{n}=\left\{y \in B_{0}^{\leq}: x_{n} \preceq y\right\}$ for any $n \geq 0$. The sequence $\left(C_{n}\right)_{n \in \mathbb{N}}$ is a decreasing sequence of nonempty $\rho$-closed convex $\rho$-bounded subsets.

Since $A_{0}^{\leq}$is $T$-invariant, then $T x_{0} \in A_{0}^{\leq}$. Using the convexity of $A_{0}^{\leq}$, one has $x_{1}=\lambda T x_{0}+(1-\lambda) x_{0} \in A_{0}^{\leq}$. Assume that $x_{n} \in A_{0}^{\leq}$, then $T x_{n} \in A_{0}^{\leq}$. Again the convexity of $A_{0}^{\leq}$, implies that $x_{n+1}=\lambda T x_{n}+(1-\lambda) x_{n} \in A_{0}^{\leq}$. Therefore, $x_{n} \in A_{0}^{\leq}$for all $n \geq 0$. Hence, for all $n \geq 0$, there exists $y \in B$ such that $x_{n} \preceq y$ and $\rho\left(x_{n}-y\right)=d_{\rho}(A, B)$. Thus, there exists $y \in B_{0}^{\preceq}$ such that $x_{n} \preceq y$. Therefore, $C_{n}$ is nonempty for all $n \geq 0$. Moreover, since $x_{n} \preceq x_{n+1}$ then $\left(C_{n}\right)_{n \in \mathbb{N}}$ is a decreasing sequence.

Let $\left(c_{p}\right)_{p \geq 0}$ be a sequence in $C_{n} \rho$-converges to $b \in B_{0}^{\leq}$. We have $c_{p} \in C_{n}$, then $c_{p}-x_{n} \in P$ and $c_{p} \in B_{0}^{\leq}$, for all $p \geq 0$. Since $B_{0}^{\leq}$is $\rho$-closed, one has $b \in B_{0}^{\leq}$. Moreover, $c_{p}-x_{n} \stackrel{\rho}{\longrightarrow} b-x_{n}$. Thus, $b-x_{n} \in P$ since $P$ is $\rho$-closed. Hence, $b \in C_{n}$. Therefore, $C_{n}$ is $\rho$-closed for all $n \geq 0$.

As $P$ and $B_{0}^{\leq}$are convex, it is easy to see that $C_{n}$ is convex for all $n \geq 0$. Furthermore, $C_{n}$ is $\rho$-bounded for all $n \geq 0$.

By Lemma $1, X_{\rho}$ satisfies the property $(R)$. Then, $C_{\infty}=$ $\cap_{n \geq 0} C_{n}$ is nonempty and $\rho$-closed convex.

Moreover, $T\left(C_{\infty}\right) \subseteq C_{\infty}$. In fact, let $z \in C_{\infty}$; then, $x_{n} \preceq z$ for all $n \geq 0$. Since $T$ is monotone and $x_{n} \preceq T x_{n}$, one has $x_{n} \preceq T z$ for all $n \geq 0$. Moreover, $T z \in B_{0}^{\preceq}$ since $B_{0}^{\preceq}$ is $T$-invariant. Hence, $T\left(C_{\infty}\right) \subseteq C_{\infty}$.

Consider the type function $\tau: C_{\infty} \longrightarrow[0,+\infty]$ generated by the sequence $\left(x_{n}\right)_{n \in \mathbb{N}}$, that is, $\tau(z)=\lim \sup _{n \longrightarrow+\infty} \rho$ $\left(x_{n}-z\right)$ for all $z \in C_{\infty}$. Let $\left(z_{p}\right)_{p \in \mathbb{N}}$ be a minimizing sequence of $\tau$. By Lemma 2, $\left(z_{p}\right)_{p} \rho$-converges to a point $y \in C_{\infty}$ since $C_{\infty}$ is $\rho$-closed. Otherwise,

$$
\begin{aligned}
\tau\left(T z_{p}\right) & =\limsup _{n \longrightarrow+\infty} \rho\left(x_{n+1}-T z_{p}\right) \\
& \leq(1-\lambda) \limsup _{n \longrightarrow+\infty} \rho\left(x_{n}-T z_{p}\right)+\lambda \limsup _{n \longrightarrow+\infty} \rho\left(x_{n}-z_{p}\right) \\
& \leq(1-\lambda) \tau\left(T z_{p}\right)+\lambda \tau\left(z_{p}\right) .
\end{aligned}
$$

Hence, $\tau\left(T z_{p}\right) \leq \tau\left(z_{p}\right)$. Then, $\left(T z_{p}\right)_{p \geq 0}$ is also a minimizing sequence of $\tau$. By Lemma 2, $\left(T z_{p}\right)_{p} \rho$-converges to $y$. As $T$ is $\rho$-continuous on $B$, then $\left(T z_{p}\right)_{p} \rho$-converges to $T y$. Therefore, $T y=y$. Then, there exists $y \in B_{0}^{\leq}$such that $T y=$ 
$y$ and $x_{n} \leq y$, for all $n \in \mathbb{N}$. By the definition of $B_{0}^{\leq}$, there exists $x \in A$ such that $x \leq y$ and $\rho(x-y)=d_{\rho}(A, B)$. In order to finish the proof, we show that $x$ is a fixed point of $T$ on $A$. We have

$$
\begin{aligned}
d_{\rho}(A, B) & \leq \rho(T x-y)=\rho(T x-T y) \\
& \leq \rho(x-y)=d_{\rho}(A, B) .
\end{aligned}
$$

Then, $\rho(T x-y)=d_{\rho}(A, B)$. Moreover,

$$
\begin{aligned}
d_{\rho}(A, B) & \leq \rho\left(\frac{T x-y}{2}+\frac{x-y}{2}\right) \\
& \leq \frac{\rho(T x-y)}{2}+\frac{\rho(x-y)}{2}=d_{\rho}(A, B) .
\end{aligned}
$$

Thus, $\rho(((T x-y)+(x-y)) / 2)=d_{\rho}(A, B)$. Since $\rho$ is (UUC2), it is (SC). Hence, $T x-y=x-y$, which implies that $T x=x$. Therefore, $(x, y)$ is a best proximity pair of the mapping $T$.

To illustrate Theorem 3, we consider the following example.

Example 1. Let $X=\mathbb{R}^{2}$, we define the modular $\rho: X \longrightarrow\left[0,+\infty\left[\right.\right.$ by $\rho(x)=\left|x_{1}\right|^{2}+\left|x_{2}\right|^{2} \quad$ for all $x=\left(x_{1}, x_{2}\right) \in \mathbb{R}^{2}$. The modular $\rho$ is convex satisfying the Fatou property and (UUC1), and $X_{\rho}$ is a $\rho$-complete modular space. Consider the $\rho$-closed convex cone $P=\left\{\left(x_{1}, x_{2}\right) \in X_{\rho}:\left(x_{1}, x_{2}\right) \geq(0,0)\right\}$. Let $A=[(-1,-2)$; $(-1,2)]$ and $B=[(1,-4) ;(1,0)]$. The pair $(A, B)$ is $\rho$-closed convex and $\rho$-bounded in $X_{\rho}$ with $d_{\rho}(A, B)=4$. Moreover, $A_{0}^{\leq}=[(-1,-2) ;(-1,0)]$ and $B_{0}^{\leq}=[(1,-2) ;(1,0)]$ are $\rho$-closed. Furthermore, we have

(i) For all $x \in[(-1,-2) ;(-1,0)]$, there exists $y \in[(1,-2) ;(1,0)]$ such that $x \leq y$

(ii) For the other cases the elements of $A$ and $B$ are not comparable

Consider the mapping $T: A \cup B \longrightarrow A \cup B$ defined by $T\left(x_{1}, x_{2}\right)= \begin{cases}(-1,0), & \text { if }\left(x_{1}, x_{2}\right) \in[(-1,-2) ;(-1,0)], \\ \left(x_{1}, x_{2}\right), & \text { if }\left(x_{1}, x_{2}\right) \in[(-1,0) ;(-1,2)],\end{cases}$

$T\left(y_{1}, y_{2}\right)=(1,0), \quad$ if $\left(y_{1}, y_{2}\right) \in B$.

The mapping $T$ is monotone noncyclic relatively $\rho$-nonexpansive $\rho$-continuous on $A \cup B$, and for $x_{0}=(-1,-2) \in A_{0}^{\leq}$we have $x_{0} \leq T x_{0}$. Then, $T$ has a best proximity pair $((1,0),(-1,0))$, where $d_{\rho}(A, B)=\rho((1,0)-(-1,0))=4$.

The following corollary is an immediate consequence of Theorem 3, and it suffices to take $A=B$; therefore, $A=A_{0}^{\leq}$ and $B=B_{0}^{\leq}$. It will be considered as a generalization of Browder and Göhde fixed point theorem for monotone $\rho$-nonexpansive mappings in modular spaces (see $[7,11,12])$. This corollary result has already been mentioned by Bin Dehaish and Khamsi (see Theorem 1) [7] in the framework of modular function spaces.
Corollary 4. Let $\rho$ be a convex modular (UUC1) satisfying the Fatou property and $X_{\rho}$ be a $\rho$-complete modular space. Let $C$ be a nonempty $\rho$-closed convex $\rho$-bounded subset of $X_{\rho}$ and $T: C \longrightarrow C$ be a monotone $\rho$-nonexpansive mapping and $\rho$-continuous. If there exists $x_{0} \in C$ such that $x_{0} \leq T\left(x_{0}\right)$, then $T$ has a fixed point.

Particular case of normed vector spaces:

\section{Remark 1}

(i) If $(X,\|\cdot\|)$ is a normed vector space, then the norm $\|\cdot\|$ is continuous and satisfies the Fatou property. Moreover, if $(X,\|\cdot\|)$ is a uniformly convex Banach space, then $(X,\|\cdot\|)$ is reflexive. Hence, it satisfies the property $(R)$.

(ii) If $(X,\|\cdot\|)$ is reflexive and $(A, B)$ is a nonempty closed and bounded pair of $X$, then $\left(A_{0}^{\leq}, B_{0}^{\leq}\right)$is also closed convex pair of $X$.

First case: if $\left(A_{0}^{\leq}, B_{0}^{\leq}\right)$is empty, then there is nothing to prove.

Second case: assume that $\left(A_{0}^{\leq}, B_{0}^{\leq}\right)$is nonempty. Let $\left(x_{n}\right)_{n}$ be a sequence in $A_{0}^{\leq}$such that $x_{n} \rho$-converges to $x \in A$. There exists a sequence $\left(y_{n}\right)_{n}$ in $B$ such that $x_{n} \leq y_{n}$ and $\left\|x_{n}-y_{n}\right\|=d(A, B)$, for all $n \geq 0$. Since $X$ is reflexive and $\left(y_{n}\right)_{n}$ is bounded, then there exists a subsequence $\left(y_{\varphi(n)}\right)_{n}$ in $B$ which converges weakly to $y \in B$. Since $\left(x_{\varphi(n)}\right)_{n}$ converges weakly to $x$, then

$$
\|x-y\| \leq \liminf _{n \longrightarrow \infty}\left\|x_{\varphi(n)}-y_{\varphi(n)}\right\| .
$$

Thus, $\|x-y\|=d(A, B)$ and $x \leq y$. Hence, $x \in A_{0}^{\leq}$. Therefore, $A_{0}^{\leq}$is closed. Using the same argument, we prove that $B_{0}^{\leq}$is also closed.

In the context of uniformly convex normed vector spaces, Theorem 3 is given as follows.

Theorem 5. Let $(A, B)$ be a nonempty convex and bounded pair of a partially ordered uniformly convex Banach space $X$ such that $B_{0}^{\leq}$nonempty and closed. Let $T: A \cup B \longrightarrow A \cup B$ be a noncyclic monotone relatively nonexpansive mapping. Assume that there exists $x_{0} \in A_{0}^{\leq}$such that $x_{0} \leq T x_{0}$. Then, $T$ has a best proximity pair.

In order to weaken the assumptions of Theorem 3, we introduce the notions of uniform continuity and uniform convexity in every direction.

Definition 10. A modular $\rho$ is said to be uniformly continuous, if for any $\varepsilon>0$ and $R>0$ there exists $\eta>0$ such that $|\rho(y)-\rho(x+y)| \leq \varepsilon$, whenever $\rho(x) \leq \eta$ and $\rho(y) \leq R$.

Definition 11. Let $\rho$ be a modular. We say that $\rho$ is uniformly convex in every direction (UCED) if for any $r>0$ and a non null $z \in X_{\rho}$, we have

$$
\delta(r, z)=\inf \left\{1-\frac{1}{r} \rho\left(x+\frac{z}{2}\right): \rho(x) \leq r, \rho(x+z) \leq r\right\}>0 .
$$


We say that $\rho$ is unique uniform convexity in every direction (UUCED) if there exists $\eta(s, z)>0$, for $s \geq 0$ and $z$ nonnull in $X_{\rho}$, such that

$$
\delta(r, z)>\eta(s, z), \text { for } r>s .
$$

The following proposition characterizes relationships between uniform convexity, uniform convexity in every direction, and strict convexity of a modular.

\section{Proposition 3}
(a) (UCi) (resp. (UUCi)) implies (UCED) (resp. (UUCED)) for $i=1,2$
(b) (UUCED) implies (UCED)
(c) (UCED) implies (SC)

Proof. It is quite easy to show (a) and (b). To prove (c), let $x$, $y \in X_{\rho}$ such that $x \neq y$. If $\rho(x) \neq \rho(y)$, there is nothing to prove. Otherwise, we assume that $\rho(x)=\rho(y)=r>0$ and we consider $z=y-x$. Hence, $\rho(x+z)=\rho(y)=r$. Since $\rho$ is (UCED) then $\delta(r, z)>0$, which implies

$$
1-\frac{1}{r} \rho\left(x+\frac{z}{2}\right) \geq \delta(r, z)>0 .
$$

Thus,

$$
\rho\left(\frac{x+y}{2}\right)=\rho\left(x+\frac{y-x}{2}\right) \leq(1-\delta(r, z)) r<r .
$$

That is, $\rho((x+y) / 2)<r=(\rho(x)+\rho(y)) / 2$.

As an example of a modular which is (UUCED) but it is not (UUC1), consider the function $\rho$ defined on $X=\mathbb{R}^{\mathbb{N}}$ by

$$
\rho(x)=\rho\left(\left(x_{n}\right)\right)=\sum_{n=1}^{\infty}\left|x_{n}\right|^{n+1} \text {. }
$$

Abdou and Khamsi [8] proved that this convex modular is (UUC2) which imply that $\rho$ is (UUCED), but it is not (UUC1).

The following lemma plays an important role in the proof of the next fixed point theorem. To prove it, we use the same ideas as those used to prove Lemma 3.5 in [7].

Lemma 3. Let $\rho$ be a convex modular uniformly continuous and (UUCED). Assume that $X_{\rho}$ satisfies the property (R). Let $C$ be a nonempty $\rho$-closed convex and $\rho$-bounded subset of $X_{\rho}$ and $K$ be a nonempty $\rho$-closed convex subset of C. Let $\left(x_{k}\right)_{k \in \mathbb{N}}$ be a sequence in $C$ and consider the $\rho$-type function $\tau: K \longrightarrow[0,+\infty]$ defined by

$$
\tau(y)=\limsup _{k \longrightarrow+\infty} \rho\left(y-x_{k}\right) .
$$

Then, $\tau$ has a unique minimum point in $K$.

Theorem 6. Let $\rho$ be a convex modular (UUCED) and is uniformly continuous. Assume that $X_{\rho}$ satisfies the property $(R)$. Let $(A, B)$ be a nonempty convex and $\rho$-bounded pair in $X_{\rho}$ such that $B_{0}^{\leq}$is nonempty and $\rho$-closed. Let $T: A \cup B \longrightarrow A \cup B$ be a monotone noncyclic relatively $\rho$-nonexpansive mapping. Assume that there exists $x_{0} \in A_{0}^{\leq}$ such that $x_{0} \preceq T x_{0}$. Then, $T$ has a best proximity pair.
Proof. Let $x_{0} \in A_{0}^{\preceq}$ such that $x_{0} \preceq T x_{0}$. Consider the sequence $\left(x_{n}\right)_{n \geq 0}$ given by $x_{n+1}=(1-\lambda) x_{n}+\lambda T x_{n}$, where $\alpha \in(0,1)$. For any $n \geq 0$, set $B_{n}=\left\{y \in B_{0}^{\leq}: x_{n} \preceq y\right\}$. Similar to Theorem 3, one has $B_{\infty}=\cap_{n \geq 0} B_{n}$ is nonempty, $\rho$-closed convex, and $T$-invariant.

Consider the type function $\tau: B_{\infty} \longrightarrow[0,+\infty]$ generated by the sequence $\left(x_{n}\right)_{n \in \mathbb{N}}$, that is, $\tau(z)=\lim \sup _{n \longrightarrow+\infty} \rho\left(x_{n}-z\right)$ for all $z \in B_{\infty}$. By Lemma 3, we know that $\tau$ has a unique minimum point $y \in B_{\infty}$. Since $T$ is monotone noncyclic relatively $\rho$-nonexpansive, we have

$$
\begin{aligned}
\tau(T(y))= & \limsup _{n} \rho\left(x_{n+1}-T(y)\right) \\
\leq & \limsup _{n}\left((1-\lambda) \rho\left(x_{n}-T(y)\right)+\lambda \rho\left(T\left(x_{n}\right)-T(y)\right)\right) \\
\leq & (1-\lambda) \limsup _{n} \rho\left(x_{n}-T(y)\right) \\
& +\lambda \limsup _{n} \rho\left(T\left(x_{n}\right)-T(y)\right) \\
\leq & (1-\lambda) \tau(T(y))+\lambda \tau(y),
\end{aligned}
$$

which implies that $\tau(T(y)) \leq \tau(y)$, since $\lambda \in(0,1)$. Therefore, $T(y)$ is also a minimum point of $\tau$ in $B_{\infty}$. The uniqueness of the minimum point of $\tau$ implies that $T(y)=y$.

Since $y \in B_{\infty}$, then $y \in B_{0}^{\leq}$. Thus, there exists $x \in A$ such that $x \preceq y$ and $\rho(x-y)=d_{\rho}(A, B)$. Using the same idea as in the proof of Theorem 3, we show that $x$ is a fixed point of $T$ on $A$. Therefore, $T x=x, \quad T y=y$, and $\rho(x-y)=d_{\rho}(A, B)$, that is, $(x, y)$ is a best proximity pair.

In the case $A=B$, therefore $A=A_{0}^{\leq}$and $B=B_{0}^{\leq}$, we obtain the following corollary. The result of this corollary has already been mentioned by Khamsi and Bin Dehaish (see Theorem 2) [7] in modular function spaces.

Corollary 7. Let $\rho$ be a convex modular (UUCED) and uniformly continuous. Assume that $X_{\rho}$ satisfies the property (R). Let $C$ be a nonempty $\rho$-closed convex and $\rho$-bounded subset of $X_{\rho}$. Let $T: C \longrightarrow C$ be a monotone $\rho$-nonexpansive mapping. If there exists $x_{0} \in C$ such that $x_{0} \preceq T\left(x_{0}\right)$, then $T$ has a fixed point.

\section{Application}

In this section, we present an application of our main results to prove the existence of the solution for nonlinear integral equations. Let $X=L^{2}([0,1], \mathbb{R})$ be the space of measurable and square-integrable functions on $[0,1]$. Recall that $X$ is a $\rho$-complete modular space, denoted $X_{\rho}$, where $\rho(f)=\int_{[0,1]}|f(t)|^{2} \mathrm{~d} t$ is a modular. Next, we consider the following integral equation:

$$
x(t)=\operatorname{sign}(x(t)) g(t)+\int_{0}^{1} F(t, s, x(s)) \mathrm{d} s, \quad t \in[0,1],
$$

where

$$
\operatorname{sign}(x(t))= \begin{cases}1, & \text { if } x(t) \geq 0 \\ -1, & \text { if } x(t)<0\end{cases}
$$


$g \in X_{\rho}$ such that $g(t) \geq 0$ for all $t \in[0,1]$ and $F:[0,1] \times$ $[0,1] \times \mathbb{R} \longrightarrow \mathbb{R}$ is measurable in both variables $t$ and $s$ for every $x$ such that

$$
\int_{0}^{1} F(t, s, x(s)) \mathrm{d} s<\infty, \quad \forall t \in[0,1] .
$$

Recall that $x \preceq y$ if and only if $y-x \in P$, where $P=\left\{x \in X_{\rho}: x(t) \geq 0\right.$ a.e. $\}$. Consider the $\rho$-closed convex subsets $A$ and $B$ of $X_{\rho}$ given by $A=\left\{x \in X_{\rho}: \gamma \preceq x \preceq \eta\right\}$ and $B=\left\{y \in X_{\rho}: \phi \preceq y \preceq \psi\right\}$ where $\gamma, \eta, \phi$, and $\psi$ are in $X_{\rho}$. Since,

$\rho(x-y) \leq \max \{\rho(\eta-\gamma)+\rho(\psi-\phi), \rho(\phi-\eta)+\rho(\psi-\gamma)\}$

$<\infty$ for all $x, y \in A \cup B$ then $(A, B)$ is $\rho$-bounded. Moreover, $A_{0}^{\leq}=\{\eta\}$ and $B_{0}^{\leq}=\{\phi\}$ are $\rho$-closed.

Theorem 8. Let the mapping $J$ be defined by

$$
J(x)(t)=\operatorname{sign}(x(t)) g(t)+\int_{0}^{1} F(t, s, x(s)) \mathrm{d} s, \quad t \in[0,1] .
$$

Assume that the following conditions are fulfilled:

$\left(c_{1}\right) \eta(t)<0<\phi(t)$, for all $t \in[0,1]$, and $\rho(\eta-\phi)>0$

$\left(c_{2}\right)\left\{\begin{array}{l}\gamma(t) \leq-g(t)+\int_{0}^{1} F(t, s, \gamma(s)) d s \\ \eta(t) \geq-g(t)+\int_{0}^{1} F(t, s, \eta(s)) d s\end{array}\right.$ and

$\left\{\begin{array}{l}\phi(t) \leq g(t)+\int_{0}^{1} F(t, s, \phi(s)) d s \\ \psi(t) \geq g(t)+\int_{0}^{1} F(t, s, \psi(s)) d s\end{array}\right.$

$\left(c_{3}\right) F$ fulfill the following monotonicity condition $0 \leq F(t, s, x(s))-F(t, s, y(s))$, for all $(x, y) \in X_{\rho}$ such that $y \preceq x$

(c $\left.c_{4}\right) \rho(J(x)-J(y)) \leq \rho(x-y)$, whenever $x, y \in A \cup B$ such that $x \preceq y$

Then, there exists $x^{*} \in A$ and $y^{*} \in B$ such that $x^{*} \preceq y^{*}$, $J\left(x^{*}\right)=x^{*}, J\left(y^{*}\right)=y^{*}$, and $\rho\left(x^{*}-y^{*}\right)=d_{\rho}(A, B)$.

Proof. $\rho$ is a (UUC1) convex modular, then $\rho$ is (UUCED). Moreover, $X_{\rho}$ satisfies the property $(\mathscr{R})$ since $X_{\rho}$ is $\rho$-complete modular space and $\rho$ is (UUC2) (from (d) of Proposition 1) and satisfies the Fatou property.

$\rho$ is uniformly continuous. Indeed, let $\varepsilon>0$ and $r>0$, and we prove that there exists $\alpha>0$ such that

$$
|\rho(y)-\rho(x+y)| \leq \varepsilon,
$$

where $\rho(x) \leq \alpha$ and $\rho(y) \leq r$. For all $t \in[0,1]$, we have $\left.|| y(t)\right|^{2}-\left.|x(t)+y(t)|^{2}|\leq| x(t)\right|^{2}+2|y(t)||x(t)|$. Then,

$$
\begin{aligned}
|\rho(y)-\rho(x+y)| & \leq \int_{[0,1]}|x(t)|^{2} \mathrm{~d} t+2 \int_{[0,1]}|y(t) \| x(t)| \mathrm{d} t \\
& \leq \rho(x)+2 \sqrt{\rho(x)} \sqrt{\rho(y)} \\
& \leq \sqrt{\rho(x)}(\sqrt{\rho(x)}+2 \sqrt{\rho(y)})
\end{aligned}
$$

If $\rho(x) \leq 1$, then

$$
\sqrt{\rho(x)}(\sqrt{\rho(x)}+2 \sqrt{\rho(y)}) \leq \sqrt{\rho(x)}(1+2 \sqrt{r}) .
$$

Since $\sqrt{\rho(x)}(1+2 \sqrt{r}) \leq \varepsilon$, this is equivalent to $\sqrt{\rho(x)} \leq \varepsilon /(2 \sqrt{r}+1)$. Therefore, $\rho(x) \leq(\varepsilon /(2 \sqrt{r}+1))^{2}$. We take $\alpha=\min \left\{1,(\varepsilon /(2 \sqrt{r}+1))^{2}\right\}$. Hence, $\rho$ is uniformly continuous.

The condition $\left(c_{2}\right)$ implies that $J(A) \subseteq A$ and $J(B) \subseteq B$. Indeed, let $x \in A$

$$
\begin{aligned}
J(x)(t)-\gamma(t) & =-g(t)+\int_{0}^{1} F(t, s, x(s)) \mathrm{d} s-\gamma(t) \\
& \geq \int_{0}^{1}(F(t, s, x(s))-F(t, s, \gamma(s))) \mathrm{d} s \geq 0 .
\end{aligned}
$$

Then, $\gamma \preceq J(x)$. In addition,

$$
\begin{aligned}
\eta(t)-J(x)(t) & =\eta(t)-\left(-g(t)+\int_{S} F(t, s, x(s)) \mathrm{d} s\right) \\
& \geq \int_{S}(F(t, s, \eta(s))-F(t, s, x(s))) \mathrm{d} s \geq 0 .
\end{aligned}
$$

Then, $J(x) \preceq \eta$. Hence, $J(A) \subseteq A$. Using the same arguments, we show that $J(B) \subseteq B$. From condition $\left(c_{1}\right)$, one has $d_{\rho}(A, B)>0$. By condition $\left(c_{3}\right)$ and since $0 \preceq g$ on $A \cup B$, we conclude that $J$ is monotone on $A \cup B$.

Consider $x_{0}=\gamma$. Condition $\left(c_{2}\right)$ implies that $x_{0} \preceq J\left(x_{0}\right)$. Hence, by Theorem 6 , there exists $x^{*} \in A$ and $y^{*} \in B$ such that $x^{*} \preceq y^{*}, J\left(x^{*}\right)=x^{*}, J\left(y^{*}\right)=y^{*}$, and $\rho\left(x^{*}-y^{*}\right)=d_{\rho}$ $(A, B)$, that is, a best proximity pair.

Example 2. Take $F(t, s, x(s))=(t s / 3) x(s)$, for all $(t, s) \in[0,1]^{2}$ and $x \in X_{\rho}$.

Consider the functions $\gamma, \eta, \phi, \psi$, and $g$ defined on $X_{\rho}$ by $\gamma(t)=-2 t, \eta(t)=-t, \phi(t)=t, \psi(t)=2 t$, and $g(t)=(8 / 9) t$, for all $t \in[0,1]$. Denote the sets $A=\left\{x \in X_{\rho}: \gamma \preceq x \preceq \eta\right\}$ and $B=\left\{y \in X_{\rho}: \phi \preceq y \preceq \psi\right\}$. Let the mapping $J: A \cup B \longrightarrow A \cup B$ defined by

$J(x)(t)=\operatorname{sign}(x(t)) g(t)+\int_{0}^{1} F(t, s, x(s)) \mathrm{d} s, \quad t \in[0,1]$.

We will check that the mapping $J$ satisfies conditions $\left(c_{1}\right)-\left(c_{4}\right)$.

$\left(c_{1}\right)$ For every $t \in[0,1]$ the inequality $\eta(t)<0<\phi(t)$ holds, and $d_{\rho}(A, B)=\rho(\phi-\eta)=\int_{0}^{1}(2 t)^{2} \mathrm{~d} t=4 / 3$.

$\left(c_{2}\right)$ It easy to see that

$$
\begin{aligned}
& \left\{\begin{array}{l}
\gamma(t) \leq-g(t)+\int_{0}^{1} F(t, s, \gamma(s)) \mathrm{d} s, \\
\eta(t) \geq-g(t)+\int_{0}^{1} F(t, s, \eta(s)) \mathrm{d} s,
\end{array}\right. \\
& \left\{\begin{array}{l}
\phi(t) \leq g(t)+\int_{0}^{1} F(t, s, \phi(s)) \mathrm{d} s, \\
\psi(t) \geq g(t)+\int_{0}^{1} F(t, s, \psi(s)) \mathrm{d} s,
\end{array}\right.
\end{aligned}
$$

then $J(A) \subset A$ and $J(B) \subset B$ 
$\left(c_{3}\right) \quad$ We have $0 \leq F(t, s, y(s))-F(t, s$, $x(s))=(t s / 3)(y(s)-x(s))$, for all $(t, s) \in[0,1]^{2}$ and $(x, y) \in X_{\rho} \times X_{\rho}$ such that $x \leq y$.

$\left(c_{4}\right)$ Let $x \in A$ and $y \in B$ such that $y \preceq x$, we have

$$
\begin{aligned}
\rho(J(y)-J(x)) & =\int_{0}^{1}\left(\int_{0}^{1}(F(t, s, y(s))-F(t, s, y(s))) \mathrm{d} s+2 g(t)\right)^{2} \mathrm{~d} t \\
& =\int_{0}^{1}(2 g(t))^{2}\left(1+\left(\frac{\int_{0}^{1}(t s / 3)(y(s)-x(s)) \mathrm{d} s}{2 g(t)}\right)\right)^{2} \mathrm{~d} t .
\end{aligned}
$$

Let $\left.\left.u_{0} \in\right] 0,1\right]$, for any $u \in \mathbb{R}$ such that $u \geq u_{o}$ we have $u_{o}(1+u) \leq u\left(1+u_{o}\right)$. Thus,

$$
\begin{aligned}
& \int_{u_{o}}^{z} u_{o}(1+u) \mathrm{d} u \leq \int_{u_{o}}^{z} u\left(1+u_{o}\right) \mathrm{d} u \\
& u_{o}\left((1+z)^{2}-\left(1+u_{o}\right)^{2}\right) \leq\left(1+u_{o}\right)\left(z^{2}-u_{o}^{2}\right) \\
& (1+z)^{2} \leq\left(1+u_{o}\right)\left(1+u_{o}+\frac{z^{2}-u_{o}^{2}}{u_{o}}\right) \\
& (1+z)^{2} \leq\left(1+u_{o}\right)\left(1+\frac{1}{u_{o}} z^{2}\right),
\end{aligned}
$$

for all $z \geq u_{0}$. Since $\quad \int_{0}^{1}(t s / 3)(y(s)-x(s)) \mathrm{d} s$ $/ 2 g(t) \geq 1 / 8$ for all $t \in[0,1]$, then

$$
\begin{aligned}
& \rho(J(y)-J(x)) \leq \int_{0}^{1}(2 g(t))^{2}\left(1+\frac{1}{8}\right) \\
& \left(1+\frac{1}{1 / 8}\left(\frac{\int_{0}^{1}(t s / 3)(y(s)-x(s)) \mathrm{d} s}{2 g(t)}\right) \mathrm{d} t\right. \\
& \leq \frac{9}{8} \int_{0}^{1}(2 g(t))^{2}\left(1+\frac{9}{32}\left(\int_{0}^{1} s(y(s)-x(s)) \mathrm{d} s\right)^{2}\right) \mathrm{d} t \\
& \leq \frac{32}{27}\left(1+\frac{9}{32}\left(\int_{0}^{1} s^{2} \mathrm{~d} s\right)\left(\int_{0}^{1}(y(s)-x(s))^{2} \mathrm{~d} s\right)\right) \\
& \leq \frac{32}{27}\left(1+\frac{3}{32} \rho(y(s)-x(s))\right) \\
& \leq \frac{8}{9} d \rho(A, B)+\frac{1}{9} \rho(y(s)-x(s)) \\
& \leq \rho(y(s)-x(s)),
\end{aligned}
$$

where $x \in A$ and $y \in B$ such that $y \preceq x$, which implies that $J$ is a monotone relatively $\rho$-nonexpansive mapping. Thus, $J(\eta)=\eta, J(\phi)=\phi$, and $\rho(\phi-\beta)=d_{\rho}(A, B)$, that is, $(\eta, \phi)$ is a best proximity pair of $J$.

\section{Conclusions}

Throughout the paper, we have discussed some best proximity pair results for the class of monotone noncyclic relatively $\rho$-nonexpansive mappings in the framework of modular spaces equipped with a partial order defined by a $\rho$-closed convex cone. Our results are extensions of several results as in relevant items from the reference section of this paper, as well as in the literature in general. In order to show the utility of our main results, we prove the existence of the solution of an integral equation which involves monotone noncyclic relatively $\rho$-nonexpansive mappinngs.

\section{Data Availability}

No data were used to support this study.

\section{Conflicts of Interest}

The authors declare that they have no conflicts of interest regarding the publication of this paper.

\section{References}

[1] H. Nakano, Modulared Semi-Ordered Linear Spaces, Maruzen Co., Tokyo, Japan, 1st edition, 1950.

[2] W. Orlicz and J. Musielak, Orlicz Spaces and Modular Spaces: Lecture Notes in Mathematics, Springer-Verlag, Berlin, Germany, 1983.

[3] A. C. M. Ran and M. C. B. Reurings, "A fixed point theorem in partially ordered sets and some applications to matrix equations," Proceedings of the American Mathematical Society, vol. 132, no. 5, pp. 1435-1443, 2003.

[4] J. J. Nieto and R. Rodríguez-López, "Contractive mapping theorems in partially ordered sets and applications to ordinary differential equations," Order, vol. 22, no. 3, pp. 223-239, 2005.

[5] M. R. Alfuraidan, M. Bachar, and M. A. Khamsi, "On monotone contraction mappings in modular function spaces," Fixed Point Theory and Applications, vol. 2015, no. 1, 2015.

[6] M. E. Gordji, F. Sajadian, Y. J. Cho, and M. Ramezani, "A fixed point theorem for quasi-contraction mappings in partially order modular spaces with application," UPB Scientific Bulletin, Series A: Applied Mathematics and Physics, vol. 76, pp. 135-146, 2014.

[7] B. A. Bin Dehaish and M. A. Khamsi, "Browder and Göhde fixed point theorem for monotone nonexpansive mappings," Fixed Point Theory and Applications, vol. 2016, no. 1, 2016.

[8] A. A. N. Abdou and M. A. Khamsi, "Fixed point theorems in modular vector spaces," The Journal of Nonlinear Sciences and Applications, vol. 10, no. 8, pp. 4046-4057, 2017.

[9] P. Kumam, "Fixed point theorems for nonexpansive mappings in modular spaces," Archivum Mathematicum, vol. 40, pp. 345-353, 2004.

[10] K. Chaira and S. Lazaiz, "Best proximity pair and fixed point results for noncyclic mappings in modular spaces," Arab Journal of Mathematical Sciences, vol. 24, no. 2, p. 147, 2018.

[11] B. A. B. Dehaish and M. A. Khamsi, "On monotone mappings in modular function spaces," Journal of Nonlinear Sciences and Applications, vol. 9, no. 8, pp. 5219-5228, 2016.

[12] F. E. Browder, "Nonexpansive nonlinear operators in a Banach space," Proceedings of the National Academy of Sciences, vol. 54, no. 4, pp. 1041-1044, 1965. 